\title{
Critical points and reaction paths characterization on a potential energy hypersurface
}

\author{
Marie-Noëlle Ramquet, Georges Dive, ${ }^{\text {a) }}$ and Dominique Dehareng \\ Centre d'Ingénierie des Protéines, Université de Liège, Institut de Chimie B6, \\ Sart-Tilman, B-4000 Liège, Belgium
}

(Received 7 April 1999; accepted 21 December 1999)

\begin{abstract}
Most of the time, the definitions of minima, saddle points or more generally order $p$ ( $p$ $=0, \ldots, n)$ critical points, do not mention the possibility of having zero Hessian eigenvalues. This feature reflects some flatness of the potential energy hypersurface in a special eigendirection which is not often taken into account. Thus, the definitions of critical points are revisited in a more general framework within this context. The concepts of bifurcation points, branching points, and valley ridge inflection points are investigated. New definitions based on the mathematical formulation of the reaction path are given and some of their properties are outlined. (C) 2000 American Institute of Physics. [S0021-9606(00)01110-7]
\end{abstract}

\section{INTRODUCTION}

In order to explore a potential energy surface, the first step is usually to locate and characterize the minima and saddle points. The surface dimension is given by the number of degrees of freedom of the molecular system and is equal to $3 N-6$ where $N$ is the number of atoms. Most of the time, since this dimension is high and since the energy function has to be computed numerically point by point, the knowledge of the potential energy surface is often limited to some local regions of chemical interest. Minima are stable equilibrium points and thus they represent the initial and final states of the transformation process. From their nature, saddle points are unstable equilibrium points and are associated with transition state structures. Minima and saddle points still have a common property: They are stationary points of the energy function. To differentiate or characterize them, Mezey, ${ }^{1}$ Müller $^{2}$ and Schlegel ${ }^{3}$ base their reasoning on the Hessian eigenvalue spectrum. But vanishing Hessian eigenvalues is not often mentioned. Zero local conical curvatures are present at the boundaries of curvature-based topologies of potential energy hypersurfaces. They are the subject of extensive works by Mezey. ${ }^{4-6} \mathrm{He}$ also details various cases of stationary points with zero Hessian eigenvalues. ${ }^{7}$

Except within the framework of symmetry, exact zero eigenvalues rarely occur. Nevertheless some potential energy surfaces are so flat that the Hessian eigenvalues are very small in magnitude. Moreover, because the gradient never reaches zero numerically, the flatness zone looks like a stationary region. This situation can be encountered for weak complexes. The problem of Jahn-Teller (and related) effect(s) will not be considered here because this implies nonadiabatic coupling between two or more electronic states. In this framework, the derivation of the energy, the gradient, and the Hessian is questionable. In this work, we are dealing

\footnotetext{
a) Author to whom correspondence should be addressed; electronic mail: gdive@ulg.ac.be
}

with only one well-defined energy surface quite decoupled from any other one.

After locating and characterizing stationary points, it is often useful to check that a particular transition state (TS) connects the desired minima. This can be done by computing the reaction path ${ }^{8-10}$ that is the union of the two steepest descent energy paths going down the potential energy surface from the TS to the adjacent minima.

Nevertheless lots of potential energy surfaces seem to possess points where the reaction path bifurcates, splits itself, crosses one or more (equivalent) paths or simply becomes unstable. These notions have partially been studied in the literature by Baker, ${ }^{11}$ Bosh, ${ }^{12}$ Schlegel ${ }^{13}$ and Valtazanos. ${ }^{14}$ They have defined some special points as bifurcation points or branching points. Valley ridge inflection points (VRI), which are very unstable points along the reaction path, are also evoked in these papers. Most of the time, the definitions given for these concepts differ and are sometimes incompatible. Baker ${ }^{11}$ and Bosh ${ }^{12}$ do not mention the necessity of having a vanishing gradient. But Valtazanos ${ }^{14}$ shows that the steepest descent path (SDP) will never bifurcate at a nonstationary point and consequently the SDP is not suitable for studying a reaction path. They also state that in general there exist no orthogonal trajectory patterns which could serve as simplified models for channel bifurcations. Bosh $^{12}$ suggests constructing an alternative route that follows the valley, not the ridge.

In an algorithmical point of view, when computing a reaction path, the calculated points never coincide exactly with the path describing the motion of the molecules or wave packets on the potential energy hypersurface. Thus when passing through or near a zone of instability and in particular a VRI, the computed path may split in two or deviate even if in theory, the SDP does not. Considering the evolution of a wave packet along a SDP, its lateral points will follow distinct SDP, and near a VRI, the wave packet could split. Moreover following Taketsugu, ${ }^{15}$ since the reaction path ob- 
viously exists with its complementary vibrational motions, the reaction channel must bifurcate at the VRI.

The first aim of the present paper is to formulate new definitions for these particular regions near the reaction path and outline their properties in the framework of a more general definition of order $p(p=0, \ldots, n)$ critical points. The second purpose of this work is to include in this general definition the occurrence of null Hessian eigenvalues.

\section{DEFINITIONS}

In the Born-Oppenheimer approximation, ${ }^{16}$ a unique potential energy surface (PES) may be assigned to any electronic state of a given molecule. Within this context, the function describing this hypersurface is denoted $E$ and depends on the nuclear coordinates. This is a well-defined and well-behaved function for most points of $\mathrm{R}^{n}$ except for those corresponding to configurations where some nuclei occupy the same position. We then may assume that there exists an open subset of $\mathrm{R}^{n}, \Omega$, wherein $E$ is at least twice continuously differentiable. This assumption is necessary since most processes for exploring a PES are based on the location of its stationary points and rely on the continuous differentiability of the gradient of $E$. So $E$ may be approximated by a Taylor expansion, i.e.,

$\forall x_{0} \in \Omega, E\left(x_{0}+D\right) \cong E\left(x_{0}\right)+\widetilde{G}\left(x_{0}\right) D+\frac{1}{2} \widetilde{D} H\left(x_{0}\right) D$,

where $G\left(x_{0}\right)$ is the gradient vector of $E$ at $x_{0}, \widetilde{G}\left(x_{0}\right)$ its transposed, and $\left[G\left(x_{0}\right)\right]_{i}=\left(\partial E / \partial x_{i}\right)\left(x_{0}\right)$, similarly $H\left(x_{0}\right)$ is the Hessian matrix of $E$ at $x_{0}$, and $\left[H\left(x_{0}\right)\right]_{i, j}$ $=\left(\partial^{2} E / \partial x_{i} \partial x_{j}\right)\left(x_{0}\right), D \in \mathbb{R}^{n}, D \approx 0$.

By definition, ${ }^{17} x_{0}$ is a minimum if and only if

$$
\exists \varepsilon>0: \forall D \in \mathbb{R}^{n}:|D|<\varepsilon, f\left(x_{0}+D\right) \geqslant f\left(x_{0}\right)
$$

and $x_{0}$ is a saddle point if and only if

$$
\exists D, \varepsilon>0: \forall|\lambda|<\varepsilon,\left\{\begin{array}{l}
f\left(x_{0}+\lambda D\right) \leqslant f\left(x_{0}\right) \\
f\left(x_{0}+\lambda D^{\perp}\right) \geqslant f\left(x_{0}\right) \forall D^{\perp} \perp D, \quad\left|D^{\perp}\right|=|D| .
\end{array}\right.
$$

More generally, $x_{0}$ will be defined as a critical point of order $p(p=0, \ldots, n)$ if and only if

$$
\exists D_{1} \perp \ldots \perp \mathrm{D}_{\mathrm{p}}, \varepsilon>0: \forall|\lambda|<\varepsilon,\left\{\begin{array}{l}
f\left(x_{0}+\lambda D_{i}\right) \leqslant f\left(x_{0}\right), \quad(i=1, \ldots, p) \\
f\left(x_{0}+\lambda D^{\perp}\right) \geqslant f\left(x_{0}\right) \forall D^{\perp} \perp D_{i}, \quad\left|D^{\perp}\right|=\left|D_{i}\right|, \quad(i=1, \ldots, p) .
\end{array}\right.
$$

This means that at a minimum, the energy function is minimum in all directions of $R^{n}$ and at a saddle point, the energy function is maximum along one direction and minimal in all orthogonal directions. More generally, a critical point of order $p(p=0, \ldots, n)$ is a point at which the energy function is maximal along $p$ directions mutually orthogonal and minimal along $n-p$ directions orthogonal to each other and to the first $p$ ones. The case where $p=0$ corresponds to a minimum. Müller ${ }^{2}$ and Schlegel $^{3,18}$ introduce minima, saddle points, and critical points of various order in this way.

According to a general mathematical terminology, the order of a critical point of a one dimensional function is determined by the first nonvanishing derivative while the index of a critical point is given by the number of strictly negative Hessian eigenvalues. For example, 0 is a fourth order critical point for the function $f: \mathbb{R} \rightarrow \mathbb{R}: x \mapsto x^{4}$ and $(0,0)$ is of index 1 for the function $f: \mathbb{R}^{2} \rightarrow \mathrm{R}:(x, y) \mapsto x^{2}-y^{2}$.

Most of the time, the "'order' of the critical point as defined by Eq. (2.4) is given by the Hessian eigenvalue spectrum and usually corresponds to the index. The quantity $p$ used in this paper is not strictly the index but the number of orthogonal directions along which the critical point is a maximum for the function considered. These two quantities are identical when there are no vanishing Hessian eigenvalues.

\section{A. Characterization of critical points}

If the Hessian eigenvalues of a critical point are all strictly positive, the point is a minimum. If, at a critical point
$\mathrm{P}$, there are $p(p=0, \ldots, n)$ strictly negative Hessian eigenvalues and $n-p$ strictly positive ones, then $\mathrm{P}$ is a critical point of order $p$ and in particular, when $p=1, \mathrm{P}$ is a saddle point. This criterion is sufficient but not necessary. If every stationary point with exactly $p$ strictly negative Hessian eigenvalues and $n-p$ strictly positive ones is a critical point of order $p$, the converse is not true. Thus, a stationary point with a zero Hessian eigenvalue and $n-1$ strictly positive ones could either be a saddle point or a minimum. Consider the following functions $f_{1}: \mathbb{R}^{2} \rightarrow \mathbb{R}:(x, y) \mapsto x^{2}-y^{2}, f_{2}: \mathbb{R}^{2}$ $\rightarrow \mathbb{R}:(x, y) \mapsto x^{2}-y^{4}$ and $f_{3}: \mathbb{R}^{2} \rightarrow \mathbb{R}:(x, y) \mapsto x^{2}+y^{4}$. Their graphs are depicted in Figs. 1(a), 1(b), and 1(c), respectively.

Figures 1(a) and 1(b) have similar shapes and from equalities (2.2) and (2.3), it can be proven that $(0,0)$ is a saddle point in the first two cases and a minimum in the third one. The Hessian eigenvalues at $(0,0)$ are $(-2,2),(0,2)$, and $(0,2)$. In the last two cases, the Hessian eigenvalue spectra are identical even if $(0,0)$ is a saddle point for $f_{2}$ and a minimum for $f_{3}$. This example confirms that a saddle point could either be characterized by one strictly negative eigenvalue and $n-1$ strictly positive ones or by $q(q=0, \ldots, n)$ zero ones and $n-q$ strictly positive ones. ${ }^{17}$ This emphasizes that there is no simple definition of a critical point of order $p$ based on its Hessian eigenvalue spectrum if there are zero eigenvalues and consequently that the notion of index is not sufficient. Thus the term "order'" will be used within the framework of this paper as the number of orthogonal directions along which the critical point is a maximum. 

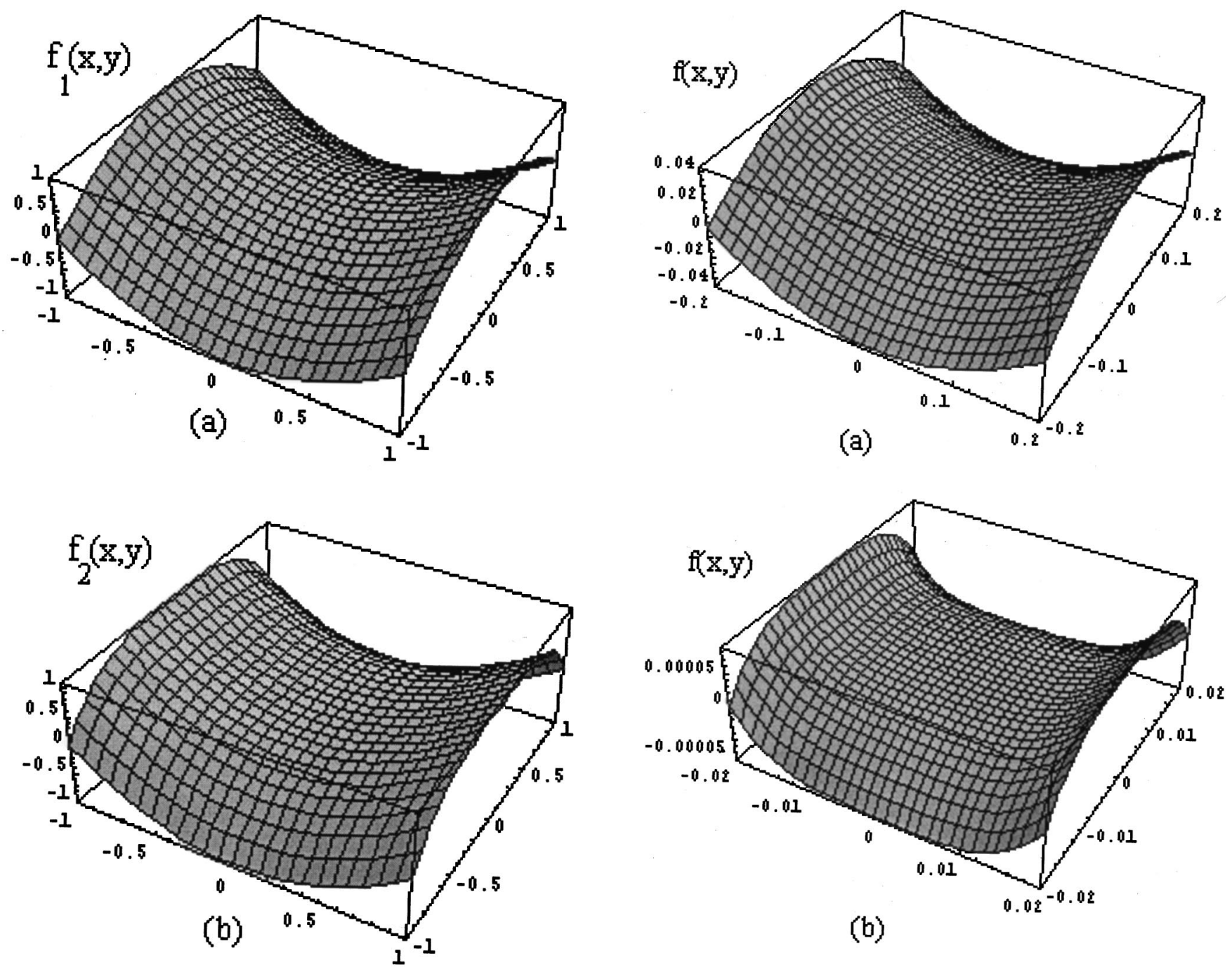

(b)

FIG. 2. Graphic of the function given in Ref. 19 at two different scales, (a)

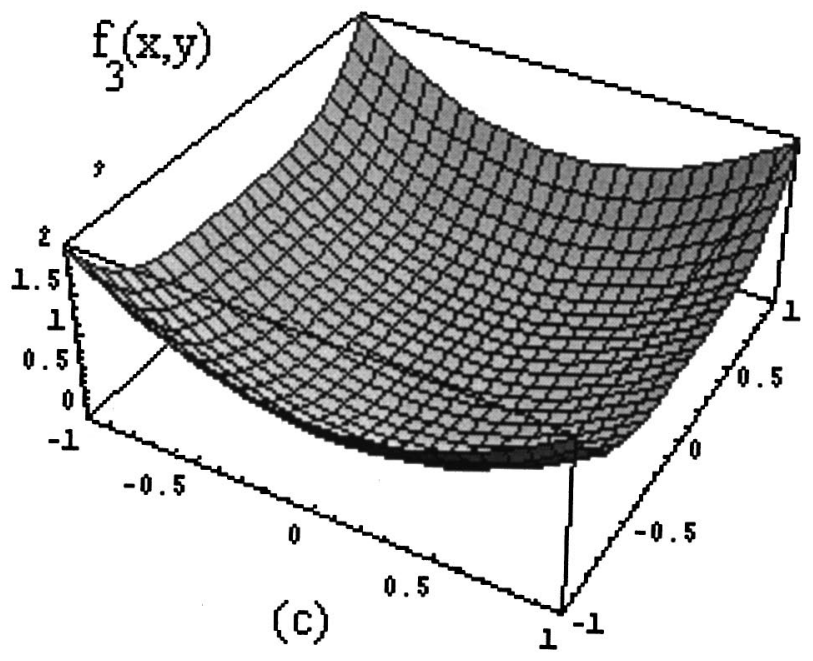

in the interval $[-0.2,0.2] \times[-0.2,0.2]$ and (b) in $[-0.02,0.02]$ $\times[-0.02,0.02]$.

farther away from it, the zero Hessian eigenvalue(s) could turn to positive or negative and even could become very large. This is illustrated by Fig. 2. The function $f$ is given in Ref. 19.

Figures 2(a) and 2(b) are two graphs of the function $f$ at different scales. The point $(0,0)$ is a critical point of order 1 , i.e., a saddle point even if the Hessian eigenvalues at $(0,0)$ are both zero. But a little bit away from it, the eigenvalues are, respectively, strictly positive and strictly negative and of large magnitude $\quad\left(\left|\mathrm{vp}_{1}(0.01,0.01)\right|=\left|\mathrm{vp}_{2}(0.01,0.01)\right|\right.$ $=0.618194)$. Depending on the scaling view, the graph of the function $f$ looks like that of $x^{2}-y^{2}$ [Fig. 2(a)] or $x^{4}$ $-y^{4}$ [Fig. 2(b)].

FIG. 1. Graphics of (a) $x^{2}-y^{2}$, (b) $x^{2}-y^{4}$, (c) $x^{2}+y^{4}$. At the point $(0,0)$, the last two functions have the same eigenvalues spectrum and they are shaped like a transition state and a minimum, respectively.

Vanishing Hessian eigenvalues simply indicate some flatness for the function graph. It is a local property and only concerns one single point, i.e., the stationary point. When vanishing eigenvalue(s) occur(s) at a critical point, a little

\section{B. Reaction path}

In any given coordinate system, the simplest method for following a reaction path from a saddle point associated with a TS down to the minima is to solve the differential equation

$$
\frac{d x(s)}{d s}=-\frac{G(x(s))}{\|G(x(s))\|}
$$




$$
x\left(s_{0}\right)=x_{0},
$$

where $\|\cdot\|$ denotes the modulus and $s$ plays the role of an arclength. $^{20-22}$

This defines the SDP; if expressed in mass weighted cartesian coordinates, this path corresponds to the intrinsic reaction path (IRC). ${ }^{8}$ This method is particularly useful if we want to go from a saddle point down to a minimum but is useless for going from below to above, i.e., for climbing along the PES. Steepest descent paths and their properties have been discussed by Mezey. ${ }^{7}$

This definition of the reaction path [Eq. (2.5)] no longer holds at stationary points since the gradient is null. The SDP is the unique solution of a Cauchy problem and is defined independently from the concepts of eigenvalues or eigenvectors. The approximated path is obtained on the basis of a second-order Taylor expansion of the energy function. In the case where there are zero eigenvalues at a stationary point, the numerical determination of the SDP requires higher order derivatives. One has to bear in mind the distinction between the analytical path, solution of Eq. (2.5), and its numerical approximation.

\section{BIFURCATION POINTS}

\section{A. Definition and properties of bifurcation points}

In Sec. III A 1, we will recall the definitions given in the literature and investigate their limitations.

\section{Point with one Hessian zero eigenvalue}

Bosch et al. ${ }^{12}$ define special points called bifurcation or branching points as points on the IRC where the curvature changes from positive to negative. i.e., points at which the IRC changes from a stable valley to an unstable ridge. They characterize them as points at which the gradient of the energy function does not necessarily vanish and where the Hessian matrix possesses one zero eigenvalue corresponding to a direction orthogonal to the tangent vector, i.e., to the vector $d x(s) / d s=-G(x(s)) /\|G(x(s))\|$. They propose a simple method for stepping at the branching point to the SDP that follows the valley. But this definition is not sufficient. Consider the function $f: \mathbb{R}^{2} \rightarrow \mathbb{R}:(x, y) \rightarrow x^{4}+(y-2)^{2}+y$. Its gradient is $\left(4 x^{3}, 2(y-2)+1\right)$ and the SDP passing through $(0,2)$ is given by the unique solution of the differential equation

$$
\begin{aligned}
& \frac{d x(s)}{d s}=-\frac{4 x(s)^{3}}{\sqrt{16 x(s)^{6}+(2 y(s)-3)^{2}}}, \\
& \frac{d y(s)}{d s}=-\frac{2 y(s)-3}{\sqrt{16 x(s)^{6}+(2 y(s)-3)^{2}}}, \\
& x(0)=0, y(0)=2,
\end{aligned}
$$

and consequently the curve $(x(s)=0, y(s)=s-2)$ is the SDP representing the reaction channel. Except for the stationary point $(0,3 / 2)$, every point of this path has a Hessian characterized by a zero eigenvalue relative to an eigenvector orthogonal to the path. They all satisfy the criteria introduced by Bosh even if no bifurcation occurs since the path $(0, s$

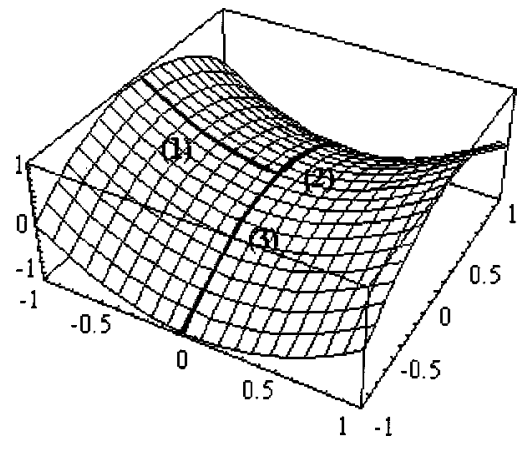

FIG. 3. Illustration of a bifurcating reaction path on the surface generated by the function $x^{2}-y^{2}$ [path (1) splits into paths (2) and (3)].

-2 ) is the only SDP passing through these points. However, most papers ${ }^{11,12}$ associating VRI with bifurcation points consider in a general sense the reaction channel or the valley path, but do not mention the steepest descent path.

A zero Hessian eigenvalue does not necessarily imply a bifurcation point and conversely, a bifurcation is not always associated with a zero Hessian eigenvalue. Consider the very well known function $f(x, y)=x^{2}-y^{2}$. The saddle point $(0,0)$ can be seen as a bifurcation point since path (1) represents an IRC that splits at $(0,0)$ into two new equivalent paths [(2) and (3)] even if no eigenvalue vanishes at $(0,0)$ (Fig. 3). In order for path (1) to be an IRC, (1) must start from an additional saddle point located above $(0,0)$.

Such an example of saddle-to-saddle descent path has been presented theoretically by Mezey in his book ${ }^{7}$ and illustrated $^{23}$ with the two-dimensional relaxed conformational map involving the two $\mathrm{C}-\mathrm{O}$ bond rotation angles of catechol.

\section{Bifurcation points within the context of symmetry}

Bifurcations are often associated with symmetry breaking. According to Baker et al., ${ }^{11}$ a bifurcation point or a branching point can be considered as a point of the reaction path where it is energetically favorable to break out the symmetry. But considering the methylamine $\operatorname{PES}^{24,25}$ allows one to conclude that this point of view is not sufficient. Figure 4 is the geometrical diagram corresponding to the PES related to the internal rotation-nitrogen inversion.

Structures Min1, Min2, and Min3 are the minima, Ts1.1, Ts1.2, Ts1.3 are saddle points while Ts 2 is a critical point of second order. Some of these paths are symmetry preserving [(1) and (2)] while the others are not [(3) and (4)]. From this point of view, Ts1.1 and Min1 are bifurcation points. But Min1 is a minimum and the question remains if it can really be treated as a bifurcation point. They are sometimes called "multibifurcation', points since they are the confluence of an infinity of SDP. ${ }^{14}$

\section{Bifurcation points as stationary points}

It has been proven ${ }^{26}$ that for the Cauchy problem [Eq. (2.5)], if there exists an open subset $\Omega$ of $\mathbb{R}^{n}$ such that (1) $f: \mathbb{R}^{n} \rightarrow \mathbb{R}^{n}: x \mapsto f(x)$ is once continuously differentiable, 


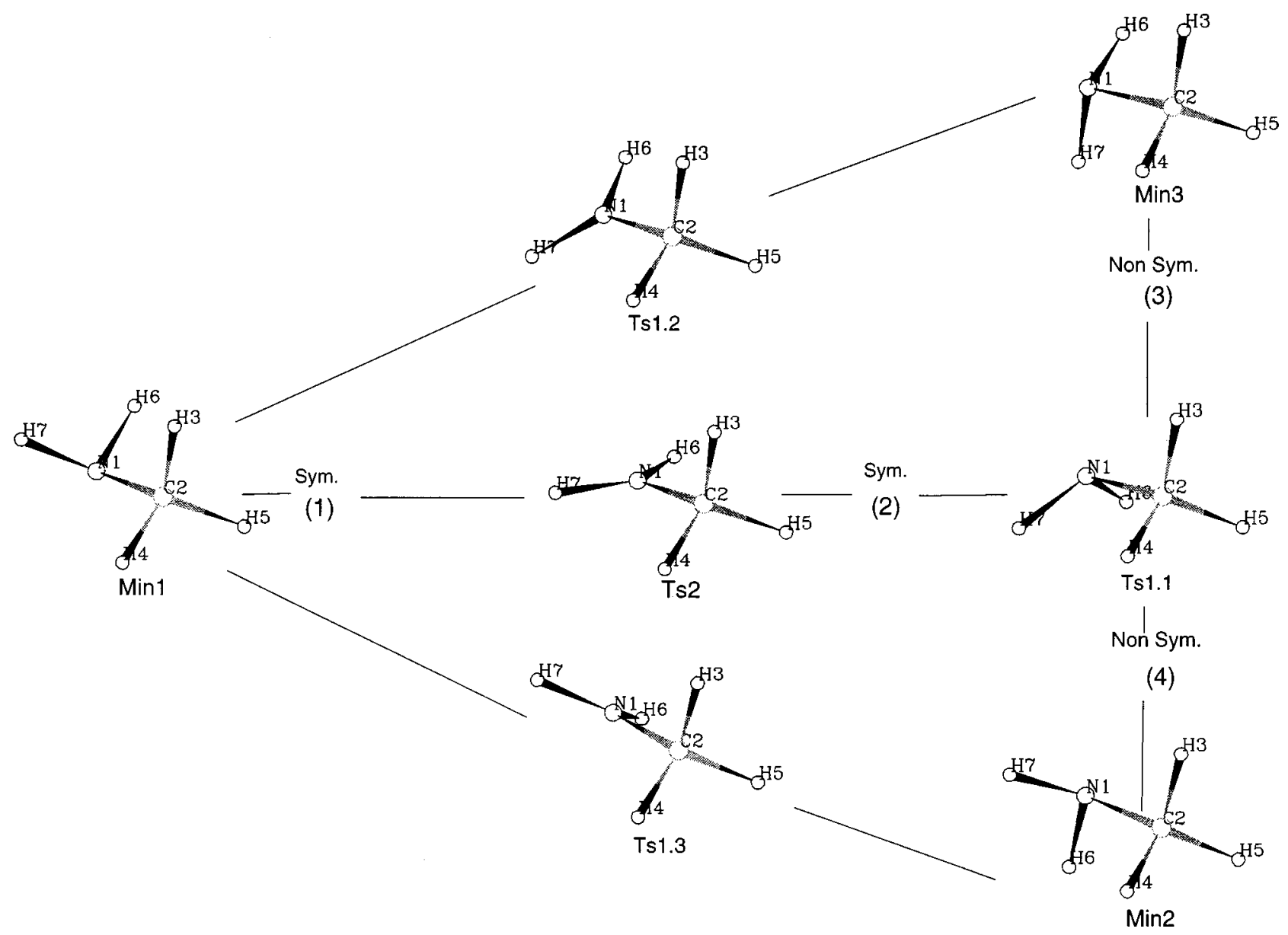

FIG. 4. Schema of a part of the methylamine PES. Three minima (Min1, Min2, Min3) are connected to each other directly through three first-order critical points (Ts1.1, Ts1.2 and Ts1.3). Min1 and Min2 or Min1 and Min3 are connected indirectly through a unique second-order critical point (Ts2).

$$
\begin{aligned}
& \forall x_{0} \in \Omega, \exists C_{1}, C_{2}: \forall x: d\left(x, x_{0}\right)<C_{1}, \\
& \left\|\left(\frac{\partial f(x)}{\partial x_{1}}, \ldots, \frac{\partial f(x)}{\partial x_{n}}\right)\right\|<C_{2},
\end{aligned}
$$

(2) the differential of $f, D f=\left(\partial f / \partial x_{1}, \ldots, \partial f / \partial x_{n}\right)$ is locally bounded in $\Omega$, then the Cauchy problem is said to be well posed and through each point of $\Omega$ passes exactly one regular SDP curve. Thus, the Cauchy problem [Eq. (2.5)] has exactly one solution $x(s)$ defined on an interval $I$ such that

(1) if $x^{\prime}(s)$ is another solution of the Cauchy problem defined on another interval $I^{\prime}$ of $\mathrm{R}$, then $\forall s \in I^{\prime}, x(s)=x^{\prime}(s)$ and $I^{\prime} \subset I$, i.e., the Cauchy problem has exactly one maximal solution,

(2) $d x(s) / d s$ is continuous within the whole domain of the definition of $x(s)$.

Since the PES was assumed to be twice continuously differentiable, the function $G(x) /\|G(x)\|$ is continuously differentiable and locally bounded on the set $\Omega$ $=\left\{x \in \mathbb{R}^{n}:\|G(x)\| \neq 0\right\}$. Consequently, at every nonstationary point of the PES passes exactly one SDP and the tangent vector is a continuous function $\left(\mathbf{v}_{\mathrm{Tr}}=d x(s) / d s\right)$; conversely, if at a point of $\Omega$ the path splits itself or if its tangent is not continuous then the point must be stationary.

Valtazanos $^{14}$ has already stated that bifurcation of SDP occurs only at stationary points. He goes on to say that be- cause of this property, SDPs are not suitable for studying the bifurcation of reaction channels. Some aspects and examples of bifurcating reaction paths have been given by Mezey. ${ }^{4,7}$

\section{B. Analytical examples}

With general analytical examples, we prove herein that the concept of bifurcation does not always have to be associated with Hessian zero eigenvalues. We first investigate $C_{1}$ cases, i.e., situations without any particular symmetry and then a general symmetrical context.

\section{General context without any symmetry}

Figure 5 represents a part of a typical model reaction path. The bifurcation point $X_{0}$ is stationary, the path joining the TS to $X_{0}$ is denoted as (1) and the two descent paths going down from $X_{0}$ are (2) and (3).

(a) if the line formed by the two paths (2) and (3) was not a broken line and formed one unique path, i.e., if the tangents to (2) and (3) ( $\mathbf{v}$ and $-\mathbf{v}$ ) with the same modulus were parallel but had opposite orientations, then no Hessian vanishing eigenvalues would be necessary at $X_{0}$.

(b) if (2) and (3) had different tangent ( $\mathbf{v}$ and $\mathbf{v}^{\prime}$ ), i.e., if they referred to two different eigenvectors and they lead to two distinct regions of the configuration space, then there would have to be at least two orthogonal descent directions 


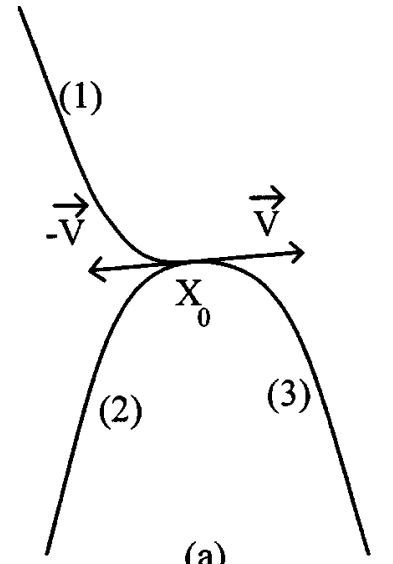

(a)

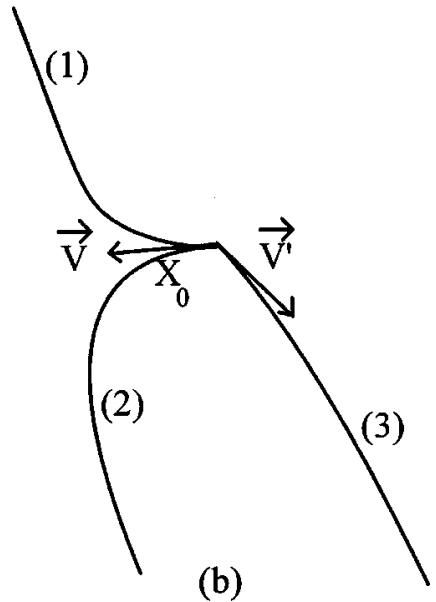

FIG. 5. Schematic illustrations of two paths bifurcating at a stationary point $x_{0}$ : (a) the paths (2) and (3) have antiparallel tangents at $x_{0}$; (b) at $x_{0}$, paths (2) and (3) tangents have different directions.

and consequently, at least two Hessian negative eigenvalues. Vanishing eigenvalues are not necessarily required.

These two examples point out that Hessian zero eigenvalues are not always necessarily required for bifurcation or path division.

\section{Particular case where there is some symmetry}

In this section, the case where the chemical system has some symmetry is investigated. We recall that symmetry breaking while following a steepest descent path for a reaction process may only occur at a stationary point and that it brings path bifurcations.

Following Pearson, ${ }^{27}$ if the symmetry is broken during a reaction process, a bifurcation must occur as there exist at least two products that are transformed into each other by some symmetry operation. Since bifurcations may only take place at stationary points, the symmetry loss may only occur at a critical point.

Mezey ${ }^{7}$ also proves that the nuclear symmetry is preserved along SDPs between critical points. Moreover he shows that if the reactant and product configurations $\left(C_{1}\right.$ and $C_{2}$ ), respectively, are not related by symmetry, then the symmetry group of the transition structure must belong to a common subgroup of the symmetry subgroup of $C_{1}$ and $C_{2}$. Various symmetry related theorems are detailed by Mezey in Refs. 28-31.

Let $x_{1}, \ldots, x_{n}$ be a complete coordinate system and $x_{1}^{\prime}, \ldots, x_{p}^{\prime}(p<n)$ a coordinate system in a restrained symmetrical context. The criterion is: a TS has the same order both in the complete coordinate system and in a symmetrical one if and only if no symmetry breaking happens. This can be intuitively understood from the following reasoning: If the orders of the stationary point in the two coordinate systems were different, i.e., if the stationary point was a minimum in the symmetrical context and a first-order critical point in the complete coordinate system, then the SDP cannot continue in symmetrical coordinates since the path reached a minimum in this context. But, in the complete coordinate system, this path still has to carry on since it only reached a first-order critical point and thus, the path has to break the symmetry. Conversely, if the symmetry is broken, some "nonsymmetrical" variables must be involved in the tangent vector and thus the critical point cannot have the same order in a symmetrical or in a complete system of coordinates.

This criterion could be used to locally reduce the dimension of the hypersurface associated with a reaction process and consequently reduce its complexity by dealing with the significant degrees of freedom only and not with the redundant ones reflecting the symmetry.

When following a reaction path, special care must be taken in the detection of bifurcation points. When reaching a stationary point, it is necessary to check out its nature, i.e., its order. Special attention must be paid when dealing with Hessian zero eigenvalues since, if at a stationary point the smallest eigenvalue is zero, it may correspond to either a minimum or a saddle point. For recall (Sec. II A) the functions $x^{2}+y^{4}$ and $x^{2}-y^{4}$ both have a zero Hessian eigenvalue and a positive one at $(0,0)$ but in the first case, $(0,0)$ is a minimum and in the second, a first-order critical point. If the Hessian is of full rank, the eigenvalues spectrum is sufficient to characterize the nature of the critical point but if the Hessian has zero eigenvalues, at least third order derivatives are required.

\section{Chemical examples}

To illustrate our considerations, we present two chemical examples already studied in the literature.

\section{Isomerization of the methoxy radical}

This example was studied earlier by Colwell et al. ${ }^{32}$ and revisited by Baker et al. ${ }^{11}$ It describes the isomerization of the methoxy radical. The interesting part of PES for our purpose is shown schematically in Fig. 6. All the unrestricted Hartree-Fock calculations have been performed in a system of internal coordinates using the STO-3G basis set.

Structures I and V correspond to the minima, II and IV are transition states since they both have an imaginary frequency. At point III, the curvature along one direction distinct from the SDP changes from positive to negative in the chosen system of internal coordinates and is a VRI. The solid curve linking I to IV through II is the minimum energy path 


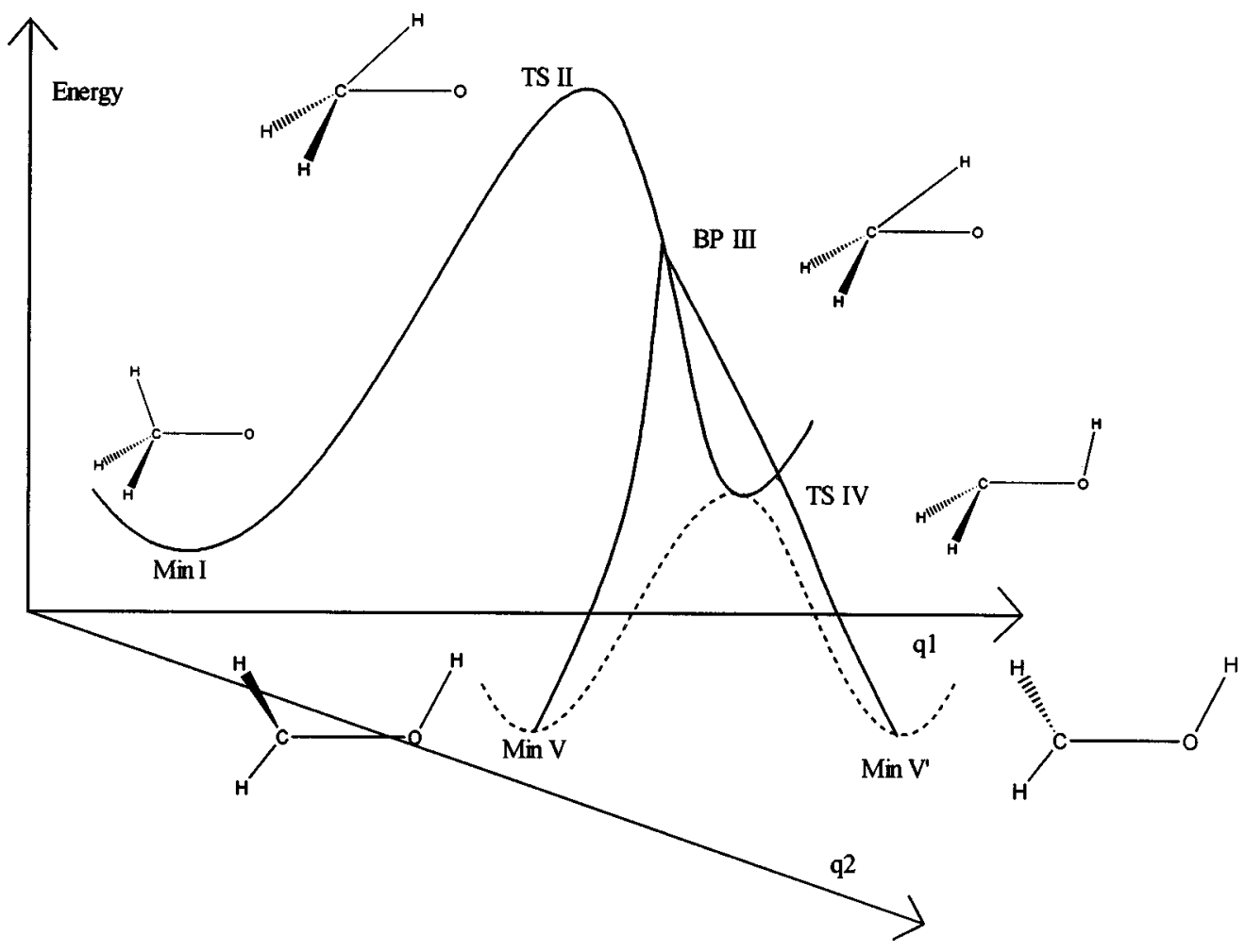

FIG. 6. Energy profile for the isomerization of the methoxy radical as described by Baker (Ref. 11).

and is symmetry preserving. Then the channel divides itself into two equivalent branches leading respectively to $V$ and $V^{\prime}$. This confirms that IV is a bifurcation point for which there are no vanishing Hessian eigenvalues. Obviously, this case of bifurcation is directly related to symmetry breaking since the reaction path leads to two structures that are transformed into each other by a reflection operation.

Moreover this example shows us how important it is to locate as many stationary points as possible since if we omit point IV then the direct path (III,V) could no longer be viewed as a SDP and consequently III would be considered as a bifurcation point instead of IV as in Baker's model ${ }^{11}$ even if III is not a stationary point. Anyway III is surrounded by a very interesting region to investigate since it is very unstable. This point is studied in Sec. IV D.

\section{Double sylation of ethylene}

Let us now consider a second example that is the subject of a reaction path study by Raaii et al. ${ }^{33}$ Their reactional schema mentioned some saddle points, minima, and a bifurcation point. Figure 7 shows some SDPs for the double sylation reaction of ethylene traced at the RHF/6-31G $(d)$ level. Once stationary points were characterized as minima or higher order critical points, the IRCs were followed from each transition state down to the corresponding minima in internal coordinates with update of the Hessian at each step. This example involves some symmetry and bifurcation points. The interesting part of the reaction scheme has six critical points (TS1, TS2, TS3, TS4, TS5.1, TS5.2) and two of them (TS5.1 and TS5.2) are transformed into each other by a reflection operation. The second order critical point (TS1) with $C_{s}$ symmetry is connected via TS4 to the mini- mum representing the products Gauche 1 and Gauche 2 that are transformed into each other by a reflection operation. These two conformers are also the endpoints of the IRC coming from TS3. Through the symmetrical TS5.1 and 5.2, the gauche minima generate the final trans structure that is a minimum. Raaii mentioned a hypothetical bifurcation point somewhere along the IRC going down from TS2. At this bifurcation point, the IRC had to split into two equivalent branches, one leading to Gauche 1 and the other one, leading to Gauche 2. Since it has been proven that bifurcations may only take place at stationary points, we propose that TS3 and TS4 are bifurcation points. From the context, these points have to be associated with symmetry breaking.

\section{ABOUT THE VRIS}

If a SDP comes to a point where the followed valley turns into a ridge, then this point is called a VRI. Such points have often been confused with bifurcation points since the region around them is unstable. When computing the path, if the actual calculated path deviates slightly from its theoretically correct route, it can diverge strongly. How fast a SDP deviates from the ridge depends on the stability of the numerical algorithm. For integrating differential equations, implicit algorithms are much more stable than explicit ones. Nevertheless, their formulation involves some circular references and they are more difficult to implement into numerical programs.

Most of the time, VRIs have been studied in the context of symmetry ${ }^{11,14,34}$ but as previously, our consideration will hold within a more general framework. The following ex- 


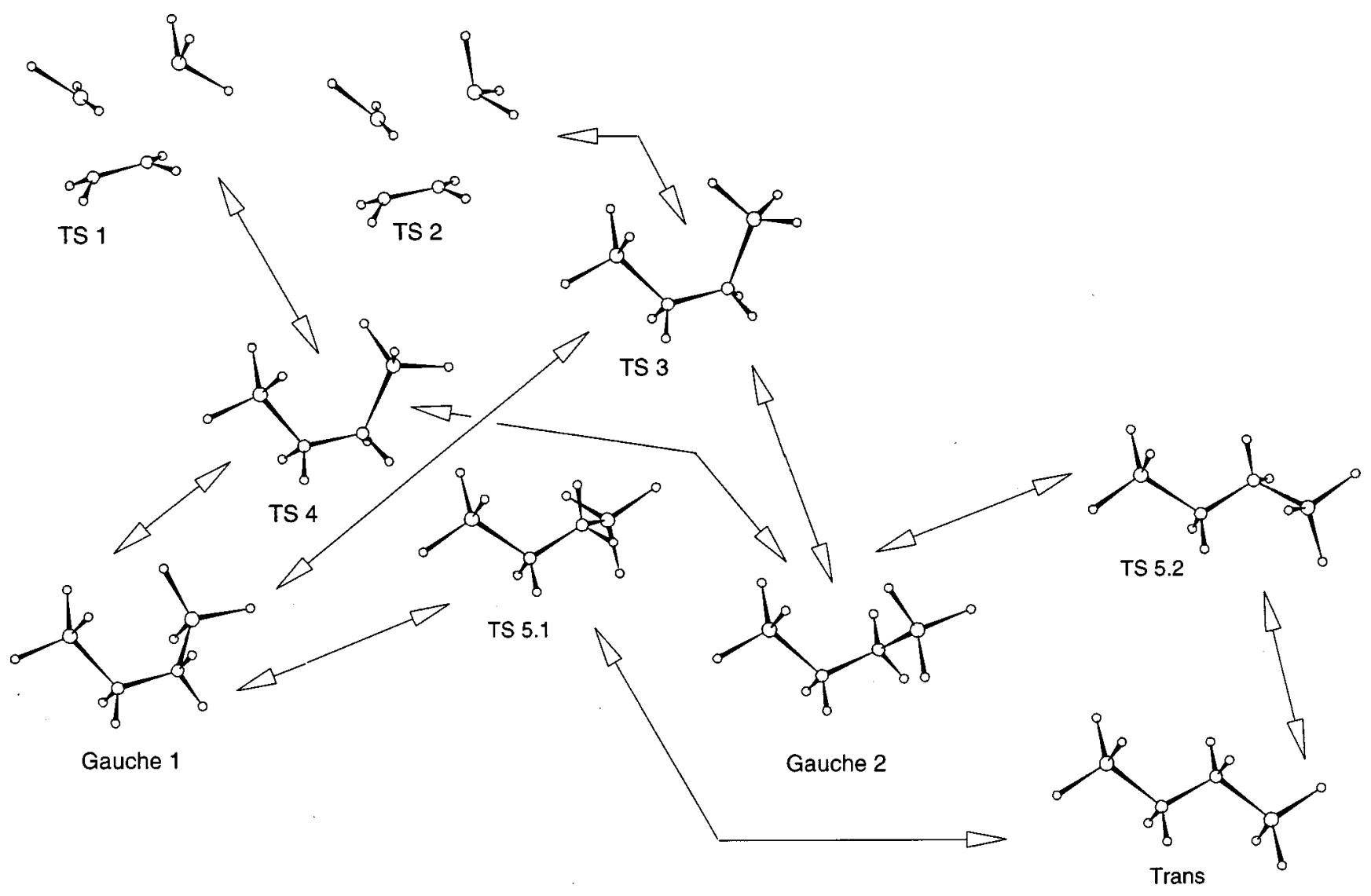

FIG. 7. Relative position of the equilibrium structures, the paths linking them and the bifurcation points.

\begin{tabular}{llr} 
& E (a.u.) & $\Delta \mathrm{E}(\mathrm{KCal})$ \\
\hline TS1 & -659.212548 & 103.508 \\
TS2 & -659.219132 & 99.376 \\
TS3 & -659.364337 & 8.259 \\
TS4 & -659.367106 & 6.521 \\
TS5.1-2 & -659.37297 & 2.841 \\
Gauche1-2 & -659.375112 & 1.497 \\
Trans & -659.377498 & 0
\end{tabular}

ample shows that a VRI must not necessarily have Hessian zero eigenvalues.

\section{A. Vanishing curvature}

Consider a PES and one of its reaction pathways. As illustrated in Fig. 8, the path is a stable valley before $x_{0}$. With Fig. 8, we prove that at $x_{0}$ there exists at least one direction different from the SDP one along which the surface has a vanishing curvature, i.e., there exists a direction $D$ orthogonal to the SDP one and such that $\widetilde{D} H\left(x_{0}\right) D=0$. The point $x_{0}$ is the VRI, $D_{1}\left(x_{0}\right)$ is the tangent vector at $x_{0}$, and $D_{2}\left(x_{0}\right)$ is the direction along which the path becomes unstable. Since at $x_{0}$, the path changes from a stable valley to an unstable ridge, there exists a direction $D_{2}\left(x_{0}\right)$ orthogonal to $D_{1}\left(x_{0}\right)$ such that $\widetilde{D}_{2}\left(x_{0}\right) H\left(x_{0}\right) D_{2}\left(x_{0}\right)=0$, i.e., the curvature vanishes.

The point $x_{0}$ must not be confused with the inflection point. From its definition, a VRI implies a vanishing curvature in a direction orthogonal to the direction of the IRC. In the case of an inflection point, a vanishing curvature occurs in the direction of the IRC.

\section{B. VRI without vanishing eigenvalues}

It is important not to confuse zero curvature and zero Hessian eigenvalue since VRI always have vanishing curvature but not necessarily vanishing Hessian eigenvalues. Figure 9 is the graph of a particular function detailed in Ref. 35 for which the point $\left(x=\frac{3}{2} \cos (\pi / 4), y=\frac{3}{2} \sin (\pi / 4)\right)$ is a VRI even if the Hessian has no vanishing eigenvalues. The curve $\gamma: \mathbb{R} \rightarrow \mathbb{R}^{2}: s \mapsto\left(\frac{3}{2} \cos (\pi / 4-s), \frac{3}{2} \sin (\pi / 4-s)\right)$ describes the SDP passing through the VRI. It is a stable valley "before", the VRI $(s \in] \pi / 4,3 \pi / 4[$ ) and an unstable ridge "after" the VRI $(s \in]-\pi / 4, \pi / 4[)$. All the calculations involved in proving that the point $\left(x=\frac{3}{2} \cos (\pi / 4), y=\frac{3}{2} \sin (\pi / 4)\right)$ is a VRI are detailed in the appendix.

Finally, the Hessian at the VRI is

$$
\left(\begin{array}{cc}
0.444 & 0 \\
0 & -0.444
\end{array}\right)
$$




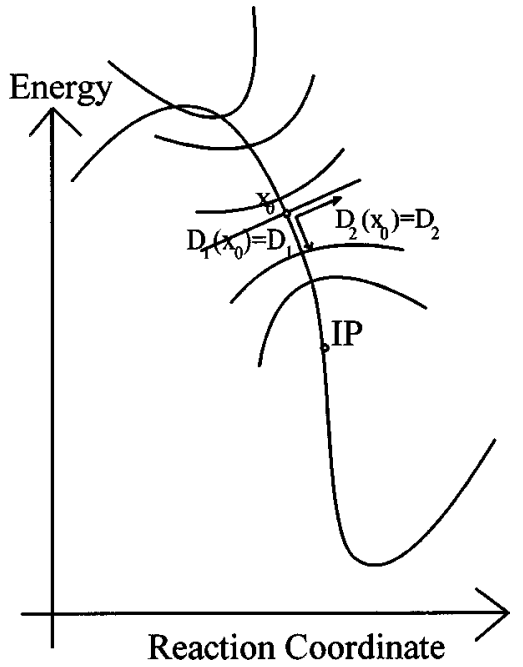

FIG. 8. Schema of a reaction path. The point $x_{0}$ is a VRI while IP is an inflection point.

and has no zero Hessian eigenvalues.

Note that this is not incompatible with what has been proven before, i.e., there exists a vanishing curvature. In this case, $\widetilde{D}=(-1,1) \Rightarrow \widetilde{D} H\left(x_{\mathrm{VRI}}\right) D=0$. This indicates that the PES is nearly flat in the direction $D$ at the VRI.

\section{Coordinate dependence of the VRI}

Let us define the matrix $M$ by $(1-g \cdot \tilde{g}) H(1-g \cdot \tilde{g})$ where $g(x)=G(x) /\|G(x)\|$.

Without loss of simplicity, we may assume that at the VRI, the gradient of the potential energy function is $(1,0, \ldots, 0)$. Thus all of its orthogonal vectors are linear combination of the vectors $\mathbf{e}_{2}=(0,1,0, \ldots, 0), \cdots, \mathbf{e}_{n}$ $=(0,0, \ldots, 0,1)$.

Since before the VRI, the IRC is stable, we have $\widetilde{D} H(x) D \geqslant 0 \forall D \perp G(x)$. After the VRI, the path is unstable so there exists an orthogonal direction $D_{1}(x)$ to $G(x)$ such

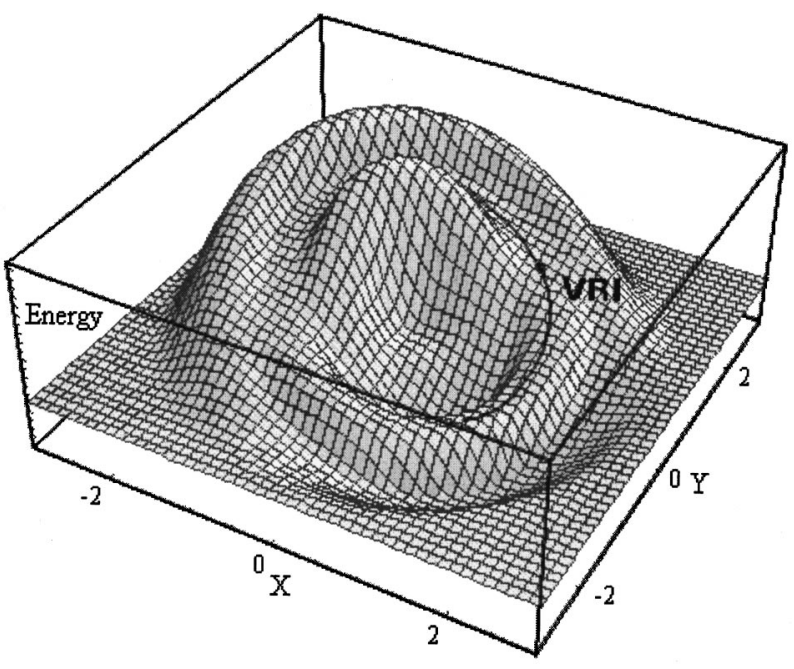

FIG. 9. Plot of the function given in Ref. 35. that $\widetilde{D}_{1}(x) H(x) D_{1}(x) \leqslant 0$ and $\widetilde{D} H(x) D \geqslant 0$ for all $D$ orthogonal to both $D_{1}(x)$ and $G(x)$. Thus, at the VRI, $\widetilde{D}_{1} H\left(x_{0}\right) D_{1}=0\left(D_{1}=D_{1}\left(x_{0}\right)\right)$.

For each $D$ orthogonal to $G\left(x_{0}\right)=g\left(x_{0}\right)=g$ $=(1,0, \ldots, 0)=\mathbf{e}_{1},\left\langle D, G\left(x_{0}\right)\right\rangle=0$ where $\langle.,$.$\rangle denotes the$ scalar product. Thus

$$
\widetilde{D}=\left\langle D, \mathbf{e}_{1}\right\rangle \mathbf{e}_{1}+\sum_{i=2}^{n}\left\langle D, \mathbf{e}_{i}\right\rangle \mathbf{e}_{i}=\sum_{i=2}^{n}\left\langle D, \mathbf{e}_{i}\right\rangle \mathbf{e}_{i}=\left(0, \widetilde{D}^{\prime}\right)
$$

where $\left[D^{\prime}\right]_{i}=\left\langle D, \mathbf{e}_{i}\right\rangle,(i=2, \ldots, n)$. First, this leads to

$$
\begin{aligned}
\widetilde{D} H D & =\left(\begin{array}{ll}
0 & \widetilde{D}^{\prime}
\end{array}\right)\left(\begin{array}{cc}
H_{1} & \widetilde{H}_{2} \\
H_{2} & H_{3}
\end{array}\right)\left(\begin{array}{c}
0 \\
D^{\prime}
\end{array}\right) \\
& =\left(\begin{array}{ll}
\widetilde{D}^{\prime} H_{2} & \widetilde{D}^{\prime} H_{3}
\end{array}\right)\left(\begin{array}{c}
0 \\
D^{\prime}
\end{array}\right)=\widetilde{D}^{\prime} H_{3} D^{\prime}
\end{aligned}
$$

and second to

$$
\begin{aligned}
& \widetilde{D} M D=\left(\begin{array}{ll}
0 & \widetilde{D}^{\prime}
\end{array}\right)(1-g \widetilde{g}) H(1-g \widetilde{g})\left(\begin{array}{c}
0 \\
D^{\prime}
\end{array}\right) \\
& =\left(\begin{array}{ll}
0 & \widetilde{D}^{\prime}
\end{array}\right)\left(\begin{array}{cc}
0 & 0 \\
0 & H_{3}
\end{array}\right)\left(\begin{array}{c}
0 \\
D^{\prime}
\end{array}\right)=\widetilde{D}^{\prime} H_{3} D^{\prime} .
\end{aligned}
$$

Consequently $\forall D \perp G\left(x_{0}\right), \widetilde{D} H\left(x_{0}\right) D=\widetilde{D} M\left(x_{0}\right) D$ and in particular $\widetilde{D}_{1} H\left(x_{0}\right) D_{1}=\widetilde{D}_{1} M D_{1}=0$. Since at $x_{0}, \widetilde{D} M D$ $\geqslant 0 \forall D \in \mathbb{R}^{n}, D_{1}$ must be an eigenvector of $M$. Thus, at a VRI, the matrix $M$ has one zero eigenvalue with $g$ as the eigenvector and another one with $D_{1}$ as the eigenvector. This is the only link between zero Hessian eigenvalues and VRI. Generally, the matrix $M$ is called the projected Hessian since it acts as if $H$ had been projected perpendicularly to the direction of the steepest descent path.

The following example shows that under a suitable coordinate change it is possible to turn an unstable path into a stable one, i.e., the notion of VRI is not univocally defined and depends on the system of coordinates.

Consider the two-dimensional function $f: \mathbb{R}^{2}$ $\rightarrow \mathrm{R}:(x, y) \mapsto-x\left(y^{2}+1\right)$ and the regular $C_{\infty}$ (indefinitely continuously differentiable) change of coordinates

$$
\left\{\begin{array} { l } 
{ u = x + \frac { y ^ { 2 } } { 2 } } \\
{ v = y }
\end{array} \Leftrightarrow \left\{\begin{array}{l}
x=u-\frac{v^{2}}{2} \\
y=v
\end{array}\right.\right.
$$

that transforms $f$ into $f^{\prime}: \mathbb{R}^{2} \rightarrow \mathbb{R}:(u, v) \mapsto\left(-u+v^{2} / 2\right)(1$ $\left.+v^{2}\right)$ and $(0,0)$ into $(0,0)$. In the first coordinate system, $(0,0)$ is a VRI while not in the second.

The nature of the stationary points is intrinsic and does not depend on the coordinate system. On the contrary, the position of the SDP joining two equilibrium structures may change. Thus, in general, a nonstationary point on a SDP, in particular a VRI, in one coordinate system might not be on the corresponding SDP in another coordinate system. However, in the chosen example, the SDP given by the path $\gamma: \mathbb{R} \rightarrow \mathbb{R}^{2}: s \mapsto(s, 0)$ is not modified under the proposed change of variables. 
(1) In both coordinate systems, the curve $\gamma: \mathbb{R} \rightarrow \mathbb{R}^{2}: s \mapsto(s, 0)$ is the SDP passing through $(0,0)$ since it is the only solution to the two Cauchy problems

$$
\left\{\begin{array} { l } 
{ \frac { d x ( s ) } { d s } = \frac { ( y ^ { 2 } ( s ) + 1 ) } { \sqrt { ( y ^ { 2 } ( s ) + 1 ) ^ { 2 } + 4 x ^ { 2 } ( s ) y ^ { 2 } ( s ) } } } \\
{ \frac { d y ( s ) } { d s } = \frac { 2 x ( s ) y ( s ) } { \sqrt { ( y ^ { 2 } ( s ) + 1 ) ^ { 2 } + 4 x ^ { 2 } ( s ) y ^ { 2 } ( s ) } } } \\
{ x ( 0 ) = 0 , y ( 0 ) = 0 }
\end{array} \text { and } \left\{\begin{array}{l}
\frac{d u(s)}{d s}=\frac{\left(1+v^{2}(s)\right)}{\sqrt{\left(1+v^{2}(s)\right)^{2}+v^{2}(s)\left(1+2 v^{2}(s)-2 u(s)\right)^{2}}} \\
\frac{d v(s)}{d s}=-\frac{v(s)\left(1+2 v^{2}(s)-2 u(s)\right)}{\sqrt{\left(1+v^{2}(s)\right)^{2}+v^{2}(s)\left(1+2 v^{2}(s)-2 u(s)\right)^{2}}} \\
u(0)=0, v(0)=0
\end{array}\right.\right.
$$

(2) In both coordinate systems, the tangent to the SDP at $(0,0)$ is $\mathbf{T}=(-1,0)$ and thus the normal is $\mathbf{N}=(0,1)$. In the first coordinate system, the path is stable before $(0,0)$ and unstable after, while in the second system the path is stable on a neighborhood before and after $(0,0)$,

$f(P+\lambda \mathbf{N})-f(P)=-x\left(\lambda^{2}+1\right)-(-x)=-x \lambda^{2}$,

$$
P=(x, 0) \in \gamma,
$$

$f^{\prime}(P+\lambda \mathbf{N})-f(P)=\left(-u+\frac{\lambda^{2}}{2}\right)\left(1+\lambda^{2}\right), \quad P=(u, 0) \in \gamma$.

Thus, the function $f(P+\lambda \mathbf{N})-f(P),(P=(x, 0))$ of the parameter $\lambda$ has a positive concavity along $\mathbf{N}$ for all negative $x$, and a negative one for the positive $x$, i.e., the SDP is stable before $(0,0)$ and unstable after. But $f^{\prime}(P+\lambda \mathbf{N})$ $-f^{\prime}(P),(P=(u, 0))$ has a positive concavity for all $u<\frac{1}{2}$, i.e., the path is stable before and after $(0,0)$.

Consequently it means that in one system of coordinates, the point $(0,0)$ is a VRI and in the other one, its image, $f(0,0)$ is not a VRI.

At $(0,0)$ and at its image $f(0,0)=(0,0)$, the projected Hessians are

$$
M=\left(\begin{array}{ll}
0 & 0 \\
0 & 0
\end{array}\right), \quad M^{\prime}=\left(\begin{array}{ll}
0 & 0 \\
0 & 1
\end{array}\right),
$$

with, respectively, 2 and 1 vanishing eigenvalues. This confirms that in the second coordinate system, $(0,0)$ cannot be a VRI since the projected Hessian has only one Hessian zero eigenvalue at $(0,0)$.

Even if we have just seen that VRI are coordinate dependent, it is still useful to locate as many of them as possible when dealing with IRCs since most of the time they involve lots of numerical problems.

\section{Numerical instability of VRI}

Even if no bifurcation can occur at a VRI unless it is also a stationary point, the vicinity region is interesting since it is very unstable. To illustrate this, consider an analytical function whose expression is given in Ref. 36 and its graphic depicted in Fig. 10.

There are four stationary points (TS, Min1, Min2, and Min2') and $(0,0)$ is the VRI. The only SDP from the TS passing through $(0,0)$ is the curve $y=0$. Clearly, $(0,0)$ is not a bifurcation point since the gradient does not vanish but the region around is very unstable.
If the calculated path deviates slightly from its symmetry preserving route because of numerical errors, then the computed path diverges from the true SDP coming from the TS. Figure 10 represents several SDPs going down, respectively, from $(0,-0.3),(0,-0.2),(0,-0.1)$, and $(0,-0.075)$ by comparison with the theoretical one passing through $(0,0)$. All of these paths are not reaction paths in the sense that they do not start from a saddle point. There is a ridge separating the two basins of attraction of Min1 and Min2 (or Min1 and Min2') but there is no TS connecting them since in this region, the surface slightly declines, i.e., for a fixed $y$ the function $f(x, y)$ is decreasing.

When computing a reaction path, bifurcations or symmetry breaking seem to occur at nonstationary points. From their nature, VRIs are very unstable points and due to rounding errors or to the algorithmic method, the computed path never coincides exactly with the theoretical SDP and consequently can deviate from its theoretical route.

\section{CONCLUSION}

In the present analysis, we have redefined and recharacterized some former concepts. If a bifurcation point is defined as a point on a reaction pathway where the IRC splits into two or more equivalent channels or for which the tangent to the path is not defined or does not exist, then these points must be critical points.

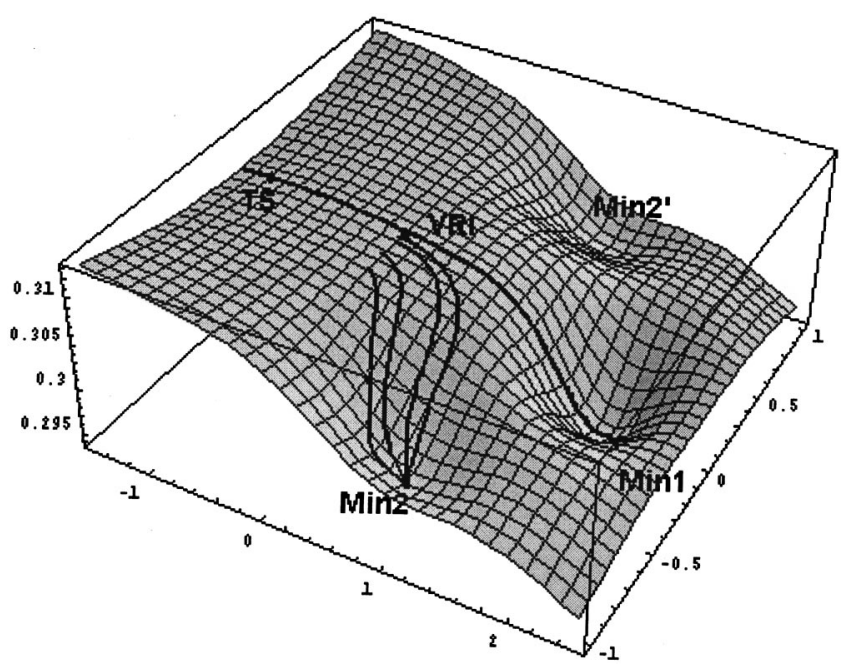

FIG. 10. Plot of the function given in Ref. 36. 
We have also restudied VRI points where the reaction pathway changes from a stable valley to an unstable ridge. We have shown that VRIs are not intrinsically defined, i.e., their location on the SDP depends on the chosen coordinate system. Moreover they are not bifurcation points unless they are also critical points.

Even if VRIs are basically not bifurcation points and do not have an intrinsic position on the IRC, they are very unstable points. If due to approximation errors, the computed path deviates slightly from its route, it may turn to another equilibrium structure.

Finally, we showed that symmetry breaking along a reaction path provokes bifurcations and that it is not necessarily characterized by zero eigenvalues for the Hessian.

\section{COMPUTATIONAL TOOLS}

All the calculations were performed with GAUSSIAN $94,{ }^{37}$ on two computers, a Dec 8400 with eight processors, and a Dec 4100 with four processors. All the investigated structures were systematically searched by full geometry optimization at the Hartree-Fock level within two basis sets, STO- $3 \mathrm{G}^{38}$ and $6-31 \mathrm{G}^{* 39}$ basis set.

\section{ACKNOWLEDGMENTS}

This work was supported in part by the Belgian program on Interuniversity Poles of Attraction initiated by the Belgian State, Prime Minister's Office, Service fédéraux des affaires scientifiques, techniques et culturelles (PAI No. P4/03), the Fonds de la Recherche Scientifique Médicale (Contract No. 3.4531.92), the pharmaceutical industrial group Servier-Adir. G.D. is chercheur qualifié of the FNRS, Brussels. M.N.R. has a grant (Ref. F 3/5/5-FC-19.007) of the FRIA, Fonds pour la formation à la recherche dans l'Industrie et dans l'agriculture.

\section{APPENDIX}

The particularity of the function introduced in Sec. IV B is to have a VRI for which there were no Hessian zero eigenvalues. This function is defined piecewise from three auxiliary functions as follows. In Cartesian coordinates:

$f$ Cart: $\mathbb{R}^{2} \rightarrow \mathbb{R}:(x, y)$

$$
\mapsto \begin{cases}f_{1}\left(\sqrt{x^{2}+y^{2}}, \frac{y-x}{\sqrt{2\left(x^{2}+y^{2}\right)}}\right) & \text { if } \sqrt{\mathrm{x}^{2}+y^{2}} \leqslant 1, \\ f_{2}\left(\sqrt{x^{2}+y^{2}}, \frac{y-x}{\sqrt{2\left(x^{2}+y^{2}\right)}}\right) & \text { if } 1<\sqrt{\mathrm{x}^{2}+y^{2}} \leqslant 2, \\ f_{3}\left(\sqrt{x^{2}+y^{2}}, \frac{y-x}{\sqrt{2\left(x^{2}+y^{2}\right)}}\right) & \text { if } 2<\sqrt{\mathrm{x}^{2}+y^{2}} \leqslant 3, \\ 1 \text { if } 3<\mathrm{x}^{2}+y^{2} & \end{cases}
$$

where

$$
f_{1}(u, v)=u^{3}(1+2 v)\left(10-15 u+6 u^{2}\right),
$$

$$
\begin{aligned}
f_{2}(u, v)= & 1+768 v\left(4.45494-4.21469 u+u^{2}\right) \\
& \times\left(2.91638-3.38143 u+u^{2}\right) \\
& \times\left(1.77208-2.61568 u+u^{2}\right) \\
& \times\left(0.81087-1.78530 u+u^{2}\right), \\
f_{3}(u, v)= & -(1+2 v)(-3+u)\left(19-21 u+6 u^{2}\right) .
\end{aligned}
$$

The point $\frac{3}{2}(\cos (\pi / 4), \sin (\pi / 4))$ is a VRI.

(1) We first note that the curve $\left(x\left(s^{\prime}\right)=\frac{3}{2} \cos (\pi / 4\right.$ $\left.\left.-\frac{2}{3} s^{\prime}\right), y\left(s^{\prime}\right)=\frac{3}{2} \sin \left(\pi / 4-\frac{2}{3} s^{\prime}\right)\right)$ is the unique solution to the Cauchy problem passing through $\frac{3}{2}(\cos (\pi / 4), \sin (\pi / 4))$.

$$
\begin{aligned}
& \frac{d x\left(s^{\prime}\right)}{d s^{\prime}}=\frac{\left[G\left(x\left(s^{\prime}\right), y\left(s^{\prime}\right)\right)\right]_{1}}{\left\|G\left(x\left(s^{\prime}\right), y\left(s^{\prime}\right)\right)\right\|}, \\
& \frac{d y\left(s^{\prime}\right)}{d s^{\prime}}=\frac{\left[G\left(x\left(s^{\prime}\right), y\left(s^{\prime}\right)\right)\right]_{2}}{\left\|G\left(x\left(s^{\prime}\right), y\left(s^{\prime}\right)\right)\right\|}, \\
& x(0)=\frac{3}{2} \cos (\pi / 4), y(0)=\frac{3}{2} \sin (\pi / 4),
\end{aligned}
$$

where $G(x, y)$ is the gradient in polar coordinates and $[G(x, y)]_{i}(i=1,2)$ is its $i$ th component.

Thus, the curve $\left(x(s)=\frac{3}{2} \cos (\pi / 4-s), y(s)=\frac{3}{2} \sin (\pi / 4\right.$ $-s)$ ) (or equivalently the curve $\left(x\left(s^{\prime}\right)=\frac{3}{2} \cos (\pi / 4\right.$ $\left.\left.\left.-\frac{2}{3} s^{\prime}\right), y\left(s^{\prime}\right)=\frac{3}{2} \sin \left(\pi / 4-\frac{2}{3} s^{\prime}\right)\right)\right)$ is the SDP passing through $\frac{3}{2}(\cos (\pi / 4), \sin (\pi / 4))$.

(2) We prove that before the VRI, the path is stable and unstable after $P \in \mathrm{SDP} \Leftrightarrow x=\frac{3}{2} \cos (\pi / 4-s), y=\frac{3}{2} \sin (\pi / 4$ $-s), s \in] 3 \pi / 4,-\pi / 4[$. The tangent vector to the SDP is $\mathbf{T}$ $=\left(\frac{3}{2} \sin (\pi / 4-s),-\frac{3}{2} \cos (\pi / 4-s)\right)$ and the orthogonal vector is thus $\mathbf{N}=\left(\frac{3}{2} \cos (\pi / 4-s), \frac{3}{2} \sin (\pi / 4-s)\right)$,

$$
\begin{aligned}
& f \operatorname{Cart}(P+\lambda \mathbf{N})-f \operatorname{Cart}(P) \\
&= f \operatorname{Cart}\left(\frac{3}{2}(1+\lambda) \cos (\pi / 4-s), \frac{3}{2}(1+\lambda) \sin (\pi / 4-s)\right) \\
& \quad-f \operatorname{Cart}\left(\frac{3}{2} \cos (\pi / 4-s), \frac{3}{2} \sin (\pi / 4-s)\right) \\
&= \lambda\left(-486-5832 \lambda^{2}-19683 \lambda^{4}\right) \sin (s) .
\end{aligned}
$$

The functions $\lambda^{4}\left(-3 \lambda^{4}+8 \lambda^{2}-6\right) \sin (s)$ and $\lambda(-486$ $\left.-5832 \lambda^{2}-19683 \lambda^{4}\right) \sin (s)$ are both decreasing if $s$ $\in] 3 \pi / 4, \pi / 4[$ and $\lambda<0$ or if $s \in] \pi / 4,-\pi / 4[$ and $\lambda>0$, and both increasing if $s \in] \pi / 4,-\pi / 4[$ and $\lambda<0$ or if $s \in] 3 \pi / 4, \pi / 4[$ and $\lambda>0$. Thus, the SDP is stable "before" $\quad \frac{3}{2}(\cos (\pi / 4), \sin (\pi / 4)) \quad$ and unstable "after" $\frac{3}{2}(\cos (\pi / 4), \sin (\pi / 4))$ and so, this point is a VRI.

${ }^{1}$ P. G. Mezey, Computational Theoretical Organic Chemistry, edited by I. G. Csizmadia and R. Daudel (Reidel, Dordrecht, 1981).

${ }^{2}$ K. Müller, Angew. Chem. Int. Ed. Engl. 19, 1 (1980).

${ }^{3}$ H. B. Schlegel, Ab Initio Methods in Quantum Chemistry-I, edited by K. P. Lawley (Wiley, New York, 1987).

${ }^{4}$ P. G. Mezey, Theor. Chim. Acta. 54, 95 (1980).

5 P. G. Mezey, Theor. Chim. Acta 62, 133 (1982).

${ }^{6}$ P. G. Mezey, Theor. Chim. Acta 67, 115 (1985).

${ }^{7}$ P. G. Mezey Potential Energy Hypersurfaces (Elsevier, New York, 1987).

${ }^{8}$ K. Fukui, Acc. Chem. Res. 14, 363 (1981).

${ }^{9}$ J. W. McIver and A. J. Komornicki, J. Am. Chem. Soc. 94, 2625 (1972).

${ }^{10}$ N. Quapp and D. Heidrich, Theor. Chim. Acta 66, 245 (1984).

${ }^{11}$ J. Baker and P. M. W. Gill, J. Comput. Chem. 9, 465 (1988).

${ }^{12}$ E. Bosch, M. Moreno, J. M. Lluch, and J. Bertrán, Chem. Phys. Lett. 160, 543 (1989).

${ }^{13}$ H. B. Schlegel, J. Chem. Soc., Faraday Trans. 90, 1569 (1994). 
${ }^{14} \mathrm{P}$. Valtazanos and K. Ruedenberg, Theor. Chim. Acta 69, 281 (1986).

${ }^{15}$ T. Taketsugu, N. Tajima, and K. Hirao, J. Chem. Phys. 105, 1933 (1996).

${ }^{16}$ M. Born, J. R. Oppenheimer, Ann. Phys. (Leipzig) 84, 457 (1927).

${ }^{17}$ M. Minoux, Programmation Mathématique (Tomes 1\&2) (Dunod, 1983).

${ }^{18}$ H. B. Schlegel, Theor. Chim. Acta 83, 15 (1992).

${ }^{19}$ The function expression used in Sec. II and depicted in Fig. 2 is given by $f: \mathbb{R}^{2} \rightarrow \mathrm{R}:(x, y) \mapsto g(x)-g(y)$,

$$
\begin{aligned}
g: \mathbb{R} \rightarrow & R: x \mapsto 1+(1+x)\left(-2+(1+x)\left(1-\left(x^{2}(1+x)^{2}(1+5 x)^{4}\right.\right.\right. \\
& \times(2+5 x)^{4}(3+5 x)^{4}(4+5 x)^{4}(1+10 x)^{4}\left(24-514 x+3865 x^{2}\right. \\
& \left.\left.\left.\left.-13375 x^{3}+23125 x^{4}-19375 x^{5}+6250 x^{6}\right)^{4}\right)\right)\right) / 1.1007531417 \mathrm{E} 11 .
\end{aligned}
$$

${ }^{20}$ K. Fukui, J. Phys. Chem. 74, 4161 (1970).

${ }^{21}$ C. Gonzales and H. B. Schlegel, J. Chem. Phys. 90, 2154 (1989).

${ }^{22}$ M. Page, C. Doubleday, and J. W. McIver, J. Chem. Phys. 93, 5634 (1990).

${ }^{23}$ P. G. Mezey, J. Am. Chem. Soc. 112, 3791 (1990)

${ }^{24}$ R. Daudel, G. Leroy, D. Peeters, and M. Sana, Quantum Chemistry (Wiley, New York, 1983).

${ }^{25}$ D. Zeroka, J. O. Jensen, and A. C. Samuels, J. Phys. Chem. A 102, 6571 (1998).

${ }^{26}$ L. Pontriaguine, Equations Différentielles Ordinaires (Collection de Moscou, Mir, 1969).

${ }^{27}$ R. G. Pearson, Acc. Chem. Res. 4, 152 (1971).

${ }^{28}$ P. G. Mezey, Int. J. Quantum Chem. 38, 699 (1990).

${ }^{29}$ P. G. Mezey, Can. J. Chem. 70, 343 (1992).

${ }^{30}$ P. G. Mezey, J. Phys. Chem. 99, 4947 (1995).

${ }^{31}$ P. G. Mezey, J. Math. Chem. 18, 133 (1995).

${ }^{32}$ S. M. Colwell, Mol. Phys. 51, 1217 (1984).

${ }^{33}$ F. Raaii and M. Gordon, J. Phys. Chem. A 102, 4666 (1998).

${ }^{34}$ W. A. Kraus and A. E. De Pristo, Theor. Chim. Acta 69, 309 (1986).

${ }^{35}$ The function used in Sec. IV B and depicted in Fig. 9 is built piecewise $f_{1}(r, a)=(1+2 a) r^{3}\left(10-15 r+6 r^{2}\right)$,

$$
\begin{aligned}
f_{2}(r, a)= & 1+14338 a-82944 a r+207360 a r^{2}-292608 a r^{3} \\
& +254976 a r^{4}-140544 a r^{5}+47872 a r^{6}-9216 a r^{7}+768 a r^{8},
\end{aligned}
$$

$$
\begin{aligned}
f_{2}(r, a)=- & (2 a+1)(r-3)^{3}\left(19-21 r+6 r^{2}\right), \\
f_{\text {Pol }}(r, t)= & f_{1}(r, \sin (t-\pi / 4)) \chi_{[0,1[}+f_{2}(r, \sin (t-\pi / 4)) \chi_{[1,2[} \\
& +f_{3}(r, \sin (t-\pi / 4)) \chi_{[2,3[}, \\
f_{\text {Cart }}(x, y)= & f_{1}\left(\sqrt{x^{2}+y^{2}}, \sin (\operatorname{Arg}(x, y)-\pi / 4)\right) \chi_{[0,1[} \\
& +f_{2}\left(\sqrt{x^{2}+y^{2}}, \sin (\operatorname{Arg}(x, y)-\pi / 4)\right) \chi_{[1,2[} \\
= & +f_{3}\left(\sqrt{x^{2}+y^{2}}, \sin (\operatorname{Arg}(x, y)-\pi / 4)\right) \chi_{[2,3[} .
\end{aligned}
$$

${ }^{36}$ The function expression in Sec. IV D and depicted in Fig. 10 is $g_{1}(x, y)=-(x+0.75)^{2}-x y^{2}$,

$$
\begin{aligned}
g_{2}(x, y) & =1-0.65 \exp \left(-x^{2}-y^{2}\right), \\
g_{3}(x, y)= & 1-0.98 \exp \left(-x^{2}-y^{2}\right), \\
g_{4}(x, y)= & \left(g_{3}(2(x-1.3), 1.5 y) g_{2}(1.5 x-0.75,1.5(y+1.6)) g_{2}\right. \\
& \times(1.5 x-0.75,1.5(y-1.6))+30) / 100,
\end{aligned}
$$$$
f(x, y)=g_{4}(x-0.39035,2 y)+g_{1}(0.5 x-0.195175, y) / 300 .
$$

${ }^{37}$ M. J. Frisch, G. W. Trucks, H. B. Schlegel, P. M. W. Gill, B. G. Johnson, M. A. Robb, J. R. Cheeseman, T. A. Keith, G. A. Petersson, J. A. Montgomery, K. Raghavachari, M. A. Al-Laham, V. G. Zakrzewski, J. V. Ortiz, J. B. Foresman, J. Cioslowski, B. B. Stefanov, A. Nanayakkara, M. Challacombe, C. Y. Peng, P. Y. Ayala, W. Chen, M. W. Wong, J. L. Andres, E. S. Replogle, R. Gomperts, R. L. Martin, D. J. Fox, J. S. Binkley, D. J. Defrees, J. Baker, J. P. Stewart, M. Head-Gordon, C. Gonzalez, and J. A. Pople, Gaussian 94, Revision D.4, Gaussian Inc., Pittsburgh, PA, 1996.

${ }^{38}$ W. J. Hehre, R. F. Stewart, and J. A. Pople, J. Chem. Phys. 51, 2657 (1969).

${ }^{39}$ T. Clark, J. Chandrasekhar, G. W. Spitznagel, and P. v. R. Shleyer, J. Comput. Chem. 4, 294 (1983). 